\title{
Commentary
}

\section{Paramedic intubation during a pandemic: Where are the consensus guidelines?}

Richard Armour BParamedPrac is an Advanced Care Paramedic ${ }^{1}$ and Sessional Academic ${ }^{2,3}$; Jennie Helmer BCom, MEd is a Paramedic Practice Leader ${ }^{1}$ and Sessional Academic ${ }^{2}$; Jon Deakin is a Paramedic Practice Leader ${ }^{1}$ and Lead Educator ${ }^{2}$

\author{
Affiliations: \\ 'British Columbia Emergency Health Services, Vancouver, Canada \\ ${ }^{2}$ The Justice Institute of British Columbia, Vancouver, Canada \\ ${ }^{3}$ Charles Sturt University, Bathurst, New South Wales
}

https://doi.org/10.33151/ajp.17.806

\section{Abstract}

There is no denying that paramedic-led intubation is a contentious issue in out-of-hospital care. Guidelines for the management of COVID-19 are developed with both patient-centred care as well as provider safety in mind, with intubation the preferred airway management strategy in patients suspected to have contracted COVID-19 requiring airway protection or invasive ventilation. However, this has re-ignited a debate which began during the Severe Acute Respiratory Syndrome (SARS) outbreak in the early 2000s around whether the benefit of paramedic-led intubation outweighs the risks to providers during a pandemic. The aim of this commentary is to revisit the evidence around paramedic-led intubation and provide a perspective on paramedic-led intubation during the COVID-19 pandemic. It is hoped this will stimulate further discussion around the benefits and risks of paramedic intubation in the setting of a pandemic.

\section{Keywords:}

paramedic; intubation; COVID-19; pandemic

Corresponding Author: Richard Armour, richard.armour@bcehs.ca 


\section{Introduction}

There is no denying that paramedic-led intubation is a contentious issue in out-of-hospital medical care, regardless of whether intubation is performed in the setting of cardiac arrest, sedation-facilitated intubation or rapid sequence intubation. In a systematic review and meta-analysis, Fouche et al (1) found that non-physicians, including paramedics, had a comparable rate of successful intubation when compared with physicians. However, this was noted to be accompanied by a generally lower first-pass success rate (1). Unfortunately, imprecision and publication bias hindered any analysis or comparison of the prevalence of adverse events between the two groups during intubation attempts (1).

Regardless of paramedic ability, research examining the patient-centred effects of out-of-hospital intubation is equivocal. Notably, AIRWAYS-2 found that intubation in out-of-hospital cardiac arrest was not associated with any improvements in survival or neurological outcome when compared with the use of an i-Gel supraglottic airway (2). This was supported by PART, which found that the use of an initial supraglottic airway was associated with a higher 72-hour survival from out-ofhospital cardiac arrest (3). However, in some settings and patient presentations it appears the provision of out-of-hospital intubation may improve outcomes (4-6). What is of particular interest in the literature is that paramedic experience with out-of-hospital intubation appears to be related not only to the success of intubation, but also patient outcomes $(5,7,8)$. Among providers with limited intubation experience mortality appears to be worsened, while in providers with significant intubation experience success and mortality appears to improve $(5,7,8)$.

But what constitutes sufficient experience for the maintenance of procedural competency in intubation? The answer is less clear. Some research suggests as few as three to five intubations per year may be sufficient for the maintenance of intubation competency $(9,10)$. However, in their most recent consensus guidelines the United Kingdom College of Paramedics recommend paramedics should be expected to perform 60 intubations before independent practice, with a subsequent expectation of two intubations per month of clinical practice (11). The heterogeneity of information related to maintenance of procedural competency is a significant confounder in the development of evidence-based policy for paramedic-led intubation.

\section{Airway management in the COVID-19 pandemic}

Guidelines for the management of patients with COVID-19 are designed not only to provide optimal patient-centred care, but also to minimise the risk to healthcare professionals. During the SARS outbreak a significant risk was identified for hospital worker nosocomial infection in those providing interventions such as intubation and positive pressure ventilation (12). This ultimately led to the debate around whether it was appropriate for paramedics to continue providing intubation during the outbreak (13). Verbreek et al argued that paramedics should not perform intubation for patients with SARS-like symptoms for two primary reasons: the complexity of personal protective equipment (PPE) removal necessitating two paramedics for appropriate doffing, and the lack of neuromuscular blocking agents in the out-of-hospital setting at the time (13). A number of respondents disagreed with the premises of Verbreek et al (13), arguing that protected intubation should remain a component of the paramedic scope of practice during a pandemic (14-17).

The landscape of out-of-hospital care has changed significantly since the SARS outbreak. The current PPE available to paramedics is more intuitive, with the same level of PPE required for intubation deployed for most patients with infectious symptoms, and a number of services now allow for paramedicled rapid sequence intubation. However, one of the largest shifts in paramedic practice is the increased use of supraglottic airways such as the i-Gel for advanced airway management in the out-of-hospital setting, particularly in cardiac arrest. These devices have the benefits of a simpler, faster insertion process with similar efficacy for the delivery of ventilation. In the setting of a pandemic, though, this must be balanced with the potential for an inferior airway seal when compared with a cuffed endotracheal tube. In the setting of the COVID-19 pandemic, a potential lack of airway seal presents a very real threat of ongoing aerosolisation during chest compressions. The airway sealing pressure of the i-Gel is reported to vary between 19 $\mathrm{cm} \mathrm{H} 2 \mathrm{O}$ and $40 \mathrm{~cm} \mathrm{H} 2 \mathrm{O}$ in patients receiving neuromuscular blockade, comparable to patients in cardiac arrest (18). Although data is sparse, it appears chest compressions during resuscitation may generate airway pressures of $40 \mathrm{~cm} \mathrm{H} 2 \mathrm{O}$ with a range between $24 \mathrm{~cm} \mathrm{H} 2 \mathrm{O}$ and $56 \mathrm{~cm} \mathrm{H} 2 \mathrm{O}$, suggesting the sealing pressure of the $\mathrm{i}-\mathrm{Gel}$ is likely exceeded during cardiac arrest $(18,19)$. What is also important to consider with the use of a supraglottic airway or the i-Gel is the potential for ongoing aerosolisation through the in-built orogastric suction ports. While this risk may be mitigated by the insertion of an orogastric tube in a closed circuit, the reduction in risk is then subsequently dependent on the quality of the filter within the suction unit.

International guidelines for emergency airway management in COVID-19 recommend the use of a 'protected' approach to intubation as the primary approach to advanced airway management (20-23). Recommendations from the epicentre of COVID-19, China, suggest that intubation is the preferred airway strategy and the use of second-generation supraglottic airways such as the i-Gel be used only to facilitate guided intubation following an initial failed attempt (20). These recommendations are supported by the Safe Airway Society (21), Difficult Airway Society, Association of Anaesthetists of the Intensive Care Society, the Faculty of Intensive Care Medicine, the Royal College of Anaesthetists (22) and the Australia and New Zealand Intensive Care Society (23). However, these guidelines infrequently reference considerations specific to 
the out-of-hospital setting, expectations for out-of-hospital management or modifications of airway management procedures for the out-of-hospital setting.

\section{The British Columbia experience}

Out-of-hospital intubation may be performed by two levels of provider in British Columbia: Advanced Care Paramedics (ACPs) and Critical Care Paramedics (CCPs). ACPs are responsible for the majority of out-of-hospital advanced airway management in British Columbia and perform intubation in the setting of cardiac arrest or using sedation-facilitated intubation with direct or video laryngoscopy. CCPs have the additional option of neuromuscular blockade and rapid sequence intubation. With concerns around aerosol generation associated with intubation and the risk of transmission from oral secretions, intubation was temporarily removed from the scope of practice for ACPs and is only allowable for CCPs using video laryngoscopy and a rapid sequence approach to intubation. Guidelines for the appropriate level of PPE during any airway management were also devised in consultation with subject matter experts, including the use of clear face shields and a level four, impervious fluid gown in addition to the N95 masks, eye protection and gloves now worn for each patient event.

This guidance was based on initial understandings of COVID-19 and the lack of consensus guidelines related to out-of-hospital care. However, as the understanding of appropriate management strategies for COVID-19 expands, it appears this guidance may now run counter to international recommendations. This is of particular concern given the potential for continued aerosolisation if an inadequate seal is obtained with the $\mathrm{i}-\mathrm{Gel}$ and is further compounded by the lack of clear guidance around the level of filter recommended within the suction unit. Important to consider as well, is the potential subsequent risk to hospital staff who may be required to perform intubation which could have been mitigated by paramedic-led intubation in the out-of-hospital setting.

\section{Current and future considerations}

Out-of-hospital emergency health services with ACPs providing intubation should consider the urgent development and dissemination of protected intubation guidelines in accordance with international recommendations and with specific reference to the challenges of the out-of-hospital setting. These guidelines should not only include guidance on the technical skill of intubation, but must also consider challenges such as the availability of appropriate equipment within the out of hospital setting for intubation and PPE requirements for providers tailored to the pathogen. Specific to the setting of outof-hospital cardiac arrest, it may be appropriate to utilise video laryngoscopy and intubation as the primary airway strategy to minimise the risk of ongoing aerosolisation in accordance with guidelines for in-hospital cardiac arrest. In emergency health services providing paramedic-led rapid sequence intubation, clear guidelines must be developed to maximise provider safety during the procedure.

\section{Conclusion}

Although first broached almost two decades ago, little progress appears to have been made with regards to consensus guidelines on the appropriateness of paramedic-led intubation and safe procedures for out-of-hospital intubation in the setting of a pandemic. Paramedic associations and medical directorates should consider the development of consensus guidelines specific to the practice of intubation in the out-ofhospital setting during a pandemic, taking into account local, national and international practices. This guidance will not only be invaluable during the current COVID-19 pandemic, but also during future pandemics.

\section{Conflict of interest}

The authors have no competing interests. Each author of this paper has completed a conflict of interest statement.

\section{References}

1. Fouche P, Stein C, Simpson P, Carlson J, Doi S. Nonphysician out-of-hospital rapid sequence intubation success and adverse events: a systematic review and meta-analysis. Ann Emerg Med 2017;70:449-59. DOI: 10.1016/j.annemergmed.2017.03.026

2. Benger J, Kirby K, Black S, et al. Effect of a strategy of a supraglottic airway device vs tracheal intubation during out of hospital cardiac arrest on functional outcome: the AIRWAYS-2 randomised clinical trial. J Am Med Assoc 2018;320:779-91. DOI: 10.1001/jama.2018.11597

3. Wang H, Schmicker R, Daya M, et al. Effect of a strategy of initial laryngeal tube insertion vs endotracheal intubation on 72-hour survival in adults with out-of-hospital cardiac arrest. ibid. 2018;320:769-78. DOI: 10.1001/jama.2018.7044

4. Bernard S, Nguyen V, Cameron P, et al. Prehospital rapid sequence intubation improves functional outcome for patients with severe traumatic brain injury: a randomised controlled trial. Ann Surg 2010;252:959-65. DOI: 10.1097/ SLA.0b013e3181efc15f

5. Pepe P, Roppolo L, Fowler R. Prehospital endotracheal intubation: elemental or detrimental? Crit Care 2015;19:121. DOI: 10.1186/s13054-015-0808-x

6. Heschi S, Meadley B, Andrew E, et al. Efficacy of pre-hospital rapid sequence intubation in paediatric traumatic brain injury: a 9-year observational study. Injury 2018;49:916-20. DOI: 10.1016/j.injury.2018.02.013

7. Wang H, Balasubramani G, Cook L, Lave J, Yealy D. Out-of-hospital endotracheal intubation experience and patient outcomes. Ann Emerg Med 2010;55:527-37. DOI: 10.1016/j.annemergmed.2009.12.020 


\section{References (continued)}

8. Bossers S, Schwarte L, Loer S, et al. Experience in prehospital endotracheal intubation significantly influences mortality of patients with severe traumatic brain injury: a systematic review and meta-analysis. PLoS One 2015;10:e0141034. DOI: 10.1371/journal.pone.0141034

9. Burton J, Baumann M, Maoz T, Bradshaw J, Lebrun J. Endotracheal intubation in a rural EMS state: procedure utilisation and impact of skills maintenance guidelines. Prehosp Emerg Care 2003;7:352-6. DOI: 10.1080/1090312090936554

10. Gillet B, Saloum D, Aghera A, Marshall J. Skill proficiency is predicted by intubation frequency of emergency medicine attending physicians. West J Emerg Med 2019;20:601-9. DOI: 10.5811/wetsjem.2019.6.42946

11. Gowens P, Aitken-Fell P, Broughton W, et al. Consensus statement: a framework for safe and effective intubation by paramedics. British Paramedic Journal 2018;3:23-7. DOI: 10 $.29045 / 14784726.2018 .06 .3 .1 .23$

12.Fowler R, Guest C, Lapinsky S, et al. Transmission of severe acute respiratory syndrome during intubation and mechanical ventilation. Am J Respir Crit Care Med 2004;169(11). DOI: 10.1164/rccm.200305-715OC

13. Verbreek R, Schwartz B, Burgess R. Should paramedics intubate patients with SARS-like symptoms? Can Med Assoc J 2003;169:299-300.

14. O'Neill R. Intubations should still be allowed with caution. ibid. 2003;169:299-300.

15. Ovens H. SARS is not unique. ibid. 2003;169:299-300.

16.Schabas R. Pre-hospital intubation and SARS. ibid.
2003;169:299-300.

17.Urszenyi, S. Discretion must reside with paramedics. ibid. 2003;169:299-300.

18.Shin H, Yoo H, Bae G, et al. Comparison of oropharyngeal leak pressure and clinical performance of LMA ProSeal and i-Gel in adults: meta-analysis and systematic review. J Int Med Res 2016;44:405-18. DOI: 10.1177/03000060515607386

19. Langhelle A, Sunde K, Wik L, Steen P. Airway pressure with chest compressions versus Heimlich manoeuvre in recently dead adults with complete airway obstruction. Resuscitation 2000;44:105-8. DOI: 10.1016/s0300-9572(00)00161-1

20.Zuo M, Huang Y, Ma W, et al. Expert recommendations for tracheal intubation in critically ill patients with novel coronavirus disease 2019. Chin Med Sci J 2020;0:10.

21.Brewster D, Chrimes N, Do T, et al. Consensus statement: Safe Airway Society principles of airway management and tracheal intubation specific to the COVID-19 adult patient group. Med J Aust 2020. Epub ahead of print. Available at: www.mja.com.au/journal/2020/consensus-statement-safeairway-society-principles-airway-management-and-tracheal

22. Cook T, El-Boghdadly K, McGuire B, et al. Consensus guidelines for managing the airway in patients with COVID-19. Anaesthesia 2020. Epub ahead of print. Available at: www.ncbi.nlm.nih.gov/pubmed/32221970 DOI: 10.1111/anae. 15054

23.Australia and New Zealand Intensive Care Society. COVID-19 Guidelines. Camberwell: ANZICS; 16 March 2020. Available at: http://cec.health.nsw.gov.au/_data/ assets/pdf_file/0004/572512/ANZICS-COVID-19-GuidelinesVersion-1.pdf 